\title{
Reluctance Actuator Characterization via FEM Simulations and Experimental Tests
}

\author{
Edgar Ramirez-Laboreo*, Carlos Sagues \\ Departamento de Informatica e Ingenieria de Sistemas (DIIS) and \\ Instituto de Investigacion en Ingenieria de Aragon (I3A), \\ Universidad de Zaragoza, Zaragoza 50018, Spain
}

\begin{abstract}
Modeling the reluctance of an electromagnetic actuator is a critical step to analyze its dynamics and design model-based controllers. On the one hand, analytical expressions based on either theoretical or empirical models often lack accuracy due to model inconsistencies. On the other, numerical methods are much more precise but require exact information about the system geometry, materials and winding configuration. In this paper we present a new method that brings together the good properties of the finite element method and of system identification techniques to obtain an accurate description of the reluctance and its derivative. Since the method is designed to identify the unknown parameters of the system, it is particularly well suited for modeling existing commercial devices. An application on a safety valve used in gas lines is included to illustrate the method and a discussion on the results shows the advantages of our proposal.
\end{abstract}

Keywords: Electromagnetic modeling, electromechanical devices, finite element analysis, inductance, reluctance, system identification

\section{Introduction}

Variable reluctance devices are currently used in many different applications. Solenoid valves are being increasingly utilized, especially in the automotive industry, e.g., in electronic stability control systems [1, or in camless engines 2, 3, and electromechanical switches can be widely found in drive-by-wire systems 4, household appliances [5], or battery chargers for electric vehicles [6], among others. In addition, novel designs of electric motors are still being investigated [7] and electromagnetic actuators are progressively replacing other types of linear-motion systems in vehicles [8] and in aeronautical applications [9] because of their accuracy, fast response, and efficiency.

Motors and linear actuators are usually designed taking into account that their motion will be eventually controlled. Thus, models are generally considered to design the geometry and select the materials that best fulfill the requirements of precision, power, efficiency, or cost [10]. On the other hand, solenoid valves and electromagnetic switches, which are commercial low-cost devices, are normally not designed to be controlled. Although having different uses, valves and switches share a common operating principle: a coil acting as an electromagnet pulls a mechanism and changes the configuration of a circuit, either a gas or liquid line or an electrical circuit. The behavior

\footnotetext{
*Corresponding author. Tel.: +34976 762472

Email addresses: ramirlab@unizar.es (Edgar Ramirez-Laboreo), csagues@unizar.es (Carlos Sagues)
}

of the electromagnetic force, which is higher the smaller the air gap, together with the absence of any type of control, causes these actuators to exhibit an on-off behavior which leads to strong impacts and wear in each operation. In this connection, several soft-landing and bounce reduction algorithms have been presented [11, 12, 13, 14, 15, many of which are based on dynamical models. The benefits are fundamentally cost-related: if the performance was improved, e.g., by increasing the service life, reducing the energy consumption or providing silent operation, these low-cost devices could replace more expensive actuators.

It is therefore evident that a dynamical model of the system is fundamental both to understand its behavior and to design control algorithms. In this regard, the most critical step when building a model of a reluctance actuator is to have a precise characterization of the reluctance. This variable depends on the geometry of the actuator and the magnetic properties of its materials, which may themselves depend on other variables such as temperature or magnetic field. It relates the magnetic flux to the electric current and, together with its derivative with respect to the gap length, is also a factor of the magnetic force that drives the motion. In other words, if both the electromagnetic dynamics and the motion of the device have to be modeled, it is imperative to obtain an expression of the reluctance as a function of the position.

The magnetic characterization of variable reluctance devices has been faced by several different approaches, ranging from purely theoretical models to more empirical 
ones. On one side, finite element method (FEM) models have been used, e.g., to optimize the design of a motor [7] or a linear actuator [16], or to calculate the attractive force of a circuit breaker [17. They have been also combined with analytical dynamical models by means of curve fitting methods [18]. A different approach is the use of magnetic equivalent circuits (MEC), a method that simplifies the description of the electromagnetic dynamics and provides an analytical expression for the reluctance. Several recent MEC-based works can be found, including [19], where a permanent magnet actuator is analyzed by means of three magnetic subcircuits, 20, where an MEC model is used to design a torque controller for a synchronous machine, or 21], where the authors propose a partially unknown reluctance which is experimentally identified. There also exist some works that use the MEC approach to calculate an analytical reluctance which is later validated or even corrected with FEM simulations [3, 22, and some papers that compare the results of both approaches [23]. Mention should also be made to the Fourier analysis approach (see, e.g., 24] and references therein). On the opposite side of the theoretical approach we find, for instance, one of the early works about contact bounce simulation [25. In that paper, the inductance of the device is simply fitted to a second order polynomial.

Despite the extensive literature on the topic, none of the proposals achieve a full and precise description of the reluctance of commercial devices. On the one hand, FEM models are accurate and allow for considering nonlinear phenomena like magnetic saturation, but some aspects of the actual system - geometry, materials, winding configuration - are usually partially or completely unknown. On the other hand, analytical expressions based on the MEC approach could be experimentally fitted to the actual device, but they have less precision because they rely on several simplifications. In this paper, we present a new modeling methodology that combines FEM simulations and experimental tests (FEM \& EXP) to obtain a precise characterization of variable reluctance devices. The proposal can be summarized in two steps: first, a complete description of the reluctance is obtained by means of an FEM model for several values of the unknown parameters, and secondly, an identification procedure determines the exact values of these parameters according to experimental data. The main contribution of the work is the method to merge both approaches, which requires only a limited amount of FEM simulations but leads to a model that is both precise and adapted to the specific device. Although the method can be used to characterize devices working under saturation, it is particularly advantageous under the assumption of magnetic linearity. Nevertheless, we will show that the results outperform those obtained with other recent approaches. As an example, it has been applied to a solenoid valve used in low-pressure gas lines. The results are discussed and the advantages of our proposal are emphasized.

\section{Theoretical fundamentals}

When describing electromechanical systems, the analysis is usually divided into the electromagnetic and the mechanical components. The dynamics of the mechanical subsystem, which is usually simpler, can be described by rigid-body models and Newton's laws of motion [21, 25] or by more sophisticated methods 23,26$]$. On the other hand, the electromagnetic part, which is the focus of this work, is generally described by two equations. First, the electrical equation of the coil,

$$
v=R i+N \frac{\mathrm{d} \phi}{\mathrm{d} t}
$$

where $v$ is the voltage across the terminals, $R$ is the resistance, $i$ is the electric current, $N$ is the number of turns and $\phi$ is the magnetic flux, and second, an equation establishing a relation between $i$ and $\phi$, which is usually given in terms of the reluctance $\mathcal{R}$ as

$$
N i=\phi \mathcal{R}
$$

As stated in the introduction, $\mathcal{R}$ depends on the materials and on the geometry of the device. When these are completely known, the MEC approach provides the following formula for its calculation

$$
\mathcal{R}=\oint \frac{\mathrm{d} l}{\mu(l) A(l)},
$$

where $l$ is the position variable that defines the closed path of the magnetic flux, i.e., the magnetic circuit, $\mu$ is the magnetic permeability and $A$ is the cross-sectional area. If the device has a complex geometry, the calculation may be simplified by partitioning the magnetic circuit into several elements of constant cross section and constant permeability. Thus, (3) is transformed into

$$
\mathcal{R}=\sum_{i} \int_{E_{i}} \frac{\mathrm{d} l}{\mu(l) A(l)} \approx \sum_{i} \frac{l_{i}}{\mu_{i} A_{i}},
$$

where $l_{i}, \mu_{i}$ and $A_{i}$ are respectively the length, the magnetic permeability and the cross section of the $i$ th element, $E_{i}$. In spite of this simplification, in many cases it may still be difficult to determine $l_{i}$ and $A_{i}$ for some of the elements, particularly for air gaps where flux fringing and leakages are always present.

A different method to determine the reluctance is by means of FEM simulations. The procedure is as follows: the simulations are firstly performed considering the electric current as the input variable, the output results are later processed to obtain the magnetic flux in the device, and, finally, the reluctance is calculated as $\mathcal{R}=N i / \phi$. The magnetic flux is by definition the integral of the magnetic flux density, B, over any cross section of the circuit,

$$
\phi=\int_{A(l)} \mathbf{B} \cdot \mathrm{d} \mathbf{s},
$$


where ds is a differential surface element vector. According to Gauss' law for magnetism, the previous expression must provide identical results when computed for any position $l$ of the circuit. However, since having a perfect description of $A(l)$ is unlikely (it covers not only the core cross section but also the surrounding air where $\mathbf{B}$ is non-zero) the applicability of (5) is very limited in practice.

Instead, the energy approach should be preferably used. The magnetic energy stored in a system is

$$
U=\int_{V}\left[\int_{-\infty}^{t} \mathbf{H} \cdot \frac{\partial \mathbf{B}}{\partial t} \mathrm{~d} t\right] \mathrm{d} v=\int_{V}\left[\int_{\mathbf{0}}^{\mathbf{B}} \mathbf{H} \cdot \delta \mathbf{B}\right] \mathrm{d} v
$$

where $V$ is the volume where the energy is computed, $\mathbf{H}$ is the magnetic field intensity, and $\mathrm{d} v$ represents a differential volume element which, under the assumptions of the MEC approach, is equal to $A(l) \mathrm{d} l$. Developing the previous expression and applying Ampère's law, i.e., $\oint \mathbf{H} \mathrm{d} l=N i$, the magnetic energy stored in an MEC can be expressed in terms of the scalar variables as

$$
U=\int_{-\infty}^{t} N i \frac{\partial \phi}{\partial t} \mathrm{~d} t=\int_{0}^{\phi} N i \delta \phi .
$$

Additionally, under the assumption that the magnetic permeability is constant, i.e., that the core material works in the linear region, the previous expressions are transformed into

$$
U=\frac{1}{2} \int_{V} \mathbf{B H} \mathrm{d} v=\frac{1}{2} N i \phi
$$

Since $U$ can be numerically computed from the results of the FEM simulations, $\phi$ can be obtained from (8) and, using (2), the expression for the reluctance is finally obtained as

$$
\mathcal{R}=\frac{(N i)^{2}}{2 U} .
$$

Either analytically or by simulations, the reluctance has to be determined for any position of the mechanism. Let $z$ be the length of the air gap of the device, which changes during its operation and, consequently, can be used as position variable. Then, the magnetic force that pulls the movable core and drives the motion is given, either in terms of $\phi$ or of $i$, by

$$
F_{\text {mag }}=-\frac{1}{2} \frac{\mathrm{d} \mathcal{R}}{\mathrm{d} z} \phi^{2}=-\frac{1}{2} \frac{N^{2}}{\mathcal{R}^{2}} \frac{\mathrm{d} \mathcal{R}}{\mathrm{d} z} i^{2}
$$

where the minus sign means that the force acts in the direction of decreasing reluctance. For a detailed explanation on how this expression is obtained see, e.g., [21.

Finally, since we will be working with both variables, recall that the relation between reluctance $\mathcal{R}$ and inductance $L$ is given by

$$
L=\frac{N^{2}}{\mathcal{R}}
$$

\section{Method description}

As stated in the introduction, the first step of our FEM \& EXP method is to build an FEM model of the system. For that, the studied device has to be inspected and all the directly measurable parameters gathered. In commercial devices, these usually include the geometry and dimensions, at least approximately, but rarely the magnetic properties of the core and the number of turns of the coil. Note that, even though in some cases this latter parameter can be determined using a winding machine, the measuring process is usually time consuming and may even lead to the destruction of the device.

The FEM model has to be parameterized in terms of the excitation current, $i$, the gap length, $z$, and also of the set of unknown parameters, $\boldsymbol{\theta}$. This set may include $N$, some geometrical dimensions and one or several parameters modeling the $\mathrm{B}-\mathrm{H}$ relation of the core material. Thus, the FEM based reluctance can be expressed as $\mathcal{R}_{\mathrm{FEM}}=\mathcal{R}_{\mathrm{FEM}}(i, z, \boldsymbol{\theta})$. If needed, additional variables such as temperature could be also incorporated to the analysis in the same way as the current. Note that if the material of the core is assumed linear, the reluctance is independent of the model excitation, i.e., $\mathcal{R}_{\mathrm{FEM}}=\mathcal{R}_{\mathrm{FEM}}(z, \boldsymbol{\theta})$, and can be calculated using (9). Considering that magnetic saturation only affects the iron core, which usually represents a small part of the reluctance, this simplification is reasonable in many cases. However, our FEM \& EXP method can also characterize systems that work under magnetic saturation, e.g., by using the two-parameter Frölich-Kennelly saturation model 21. In that case, (9) would be no longer valid and $\mathcal{R}_{\text {FEM }}$ would have to be obtained by another method.

After that, a parametric sweep has to be performed to obtain a finite set of FEM reluctance values,

$$
\Psi_{\mathrm{FEM}}=\left\{\mathcal{R}_{\mathrm{FEM}}(i, z, \boldsymbol{\theta}) \mid i \in \mathcal{I}_{\mathrm{FEM}}, z \in \mathcal{Z}_{\mathrm{FEM}}, \boldsymbol{\theta} \in \boldsymbol{\Theta}_{\mathrm{FEM}}\right\},
$$

where $\mathcal{I}_{\mathrm{FEM}}, \mathcal{Z}_{\mathrm{FEM}}$ and $\boldsymbol{\Theta}_{\mathrm{FEM}}$ are respectively the sets of discrete values of $i, z$ and $\boldsymbol{\theta}$ for which the reluctance is computed. Given that this variable is a smooth function with respect to most of the parameters, we propose to estimate $\mathcal{R}_{\mathrm{FEM}}$ at any other point using cubic spline interpolation,

$$
\mathcal{R}_{\mathrm{FEM}}(i, z, \boldsymbol{\theta}) \approx \hat{\mathcal{R}}_{\mathrm{FEM}}(i, z, \boldsymbol{\theta})=\mathrm{f}_{\mathrm{spline}}\left(\Psi_{\mathrm{FEM}}, i, z, \boldsymbol{\theta}\right),
$$

where $\hat{\mathcal{R}}$ is the estimation and $\mathrm{f}_{\text {spline }}$ is the interpolating function. It is therefore evident that the selection of $\mathcal{I}_{\text {FEM }}$, $\mathcal{Z}_{\mathrm{FEM}}$ and $\boldsymbol{\Theta}_{\mathrm{FEM}}$ is a key aspect of the method. On the one hand, $\mathcal{I}_{\mathrm{FEM}}$ and $\mathcal{Z}_{\mathrm{FEM}}$ must be selected considering the minimum and maximum values of $i$ and $z$ which will be evaluated during the experimental tests. In turn, these should be based on the physical limitations of the actuator. On the other hand, $\boldsymbol{\Theta}_{\mathrm{FEM}}$ should be centered around a nominal value of $\boldsymbol{\theta}$ and cover all possible values of the parameters. In this regard, setting reasonable bounds to geometric dimensions or magnetic properties should not be a problem because these are always limited by physics. The number of elements in each set is a trade-off between 
simulation time and model accuracy: a coarse selection of points would greatly reduce the number of FEM simulations, but a finer one would necessarily lead to better estimations of $\mathcal{R}_{\mathrm{FEM}}$. In any case, note that the reluctance calculation can be made independent of $N$ because any constant product of $N$ and $i$ will lead to the same $\mathcal{R}_{\mathrm{FEM}}$. Thus, this variable does not need to be included in the sweep. Once the reluctance is obtained, the FEM based inductance can be computed by (11) as $L_{\mathrm{FEM}}(i, z, \boldsymbol{\theta})=N^{2} / \hat{\mathcal{R}}_{\mathrm{FEM}}(i, z, \boldsymbol{\theta})$.

The values of the unknown parameters are then determined in the second step of the method. For that, experimental tests have to be firstly performed. Our proposal is to measure the position-dependent inductance of the device by placing the armature at several static positions between the minimum and maximum possible values of the gap length. If the aim of the model is not to characterize a single system, but the average behavior of a mass production device, several units of the actuator may be evaluated in order to minimize the effects of the manufacturing variability. The inductance may be measured by two different approaches. First, if an impedance analyzer is available, direct measurements can be performed using the series resistance-inductance (RL) circuit model. Otherwise, a classic linear system identification process [27] needs to be performed for every position of the device. The idea is to use a voltage step as input and record the dynamic response of the electric current. Then, each data set is used to fit a series RL model, i.e., the following dynamic equation,

$$
v=R i+L \frac{\mathrm{d} i}{\mathrm{~d} t},
$$

by minimizing the root-mean-square error (RMSE) between the simulated and the experimental values of $i$. If the FEM model depends on the excitation, several smallsignal step response tests around different levels of $i$ have to be performed.

One method or the other, the result of the process is a set of experimental values of resistance, $R_{\operatorname{EXP} \alpha}\left(i_{\beta}, z_{\gamma}\right)$, and inductance, $L_{\mathrm{EXP} \alpha}\left(i_{\beta}, z_{\gamma}\right)$, for $\alpha=1, \ldots, n, \beta=1, \ldots, m$, and $\gamma=1, \ldots, p$, where $n, m$ and $p$ are respectively the number of actuators, excitation levels and positions evaluated. Note that although the coil resistance does not depend on the position of the armature, it should not be assumed constant because it could vary between tests due to temperature changes. Hence, identifying both parameters, instead of only $L$, prevents from errors due to variations in temperature. An estimation error of the model at the $\beta$ th excitation level and $\gamma$-th position with respect to the measurements of the $\alpha$-th actuator may be defined as

$$
e_{\alpha, \beta, \gamma}(\boldsymbol{\theta})=L_{\mathrm{FEM}}\left(i_{\beta}, z_{\gamma}, \boldsymbol{\theta}\right)-L_{\mathrm{EXP} \alpha}\left(i_{\beta}, z_{\gamma}\right),
$$

and thus the sum of squared errors may be used as a performance index of the model,

$$
J(\boldsymbol{\theta})=\sum_{\alpha=1}^{n} \sum_{\beta=1}^{m} \sum_{\gamma=1}^{p}\left(e_{\alpha, \beta, \gamma}(\boldsymbol{\theta})\right)^{2} .
$$

The best-fitting parameter set is therefore the one that minimizes $J$,

$$
\boldsymbol{\theta}^{*}=\underset{\boldsymbol{\theta}}{\arg \min } J(\boldsymbol{\theta}),
$$

and the FEM \& EXP reluctance is

$$
\mathcal{R}_{\mathrm{FEM} \mathrm{\&} \mathrm{EXP}}(i, z)=\hat{\mathcal{R}}_{\mathrm{FEM}}\left(i, z, \boldsymbol{\theta}^{*}\right) .
$$

To sum up, the flow chart of the proposal is shown in Fig. 1.

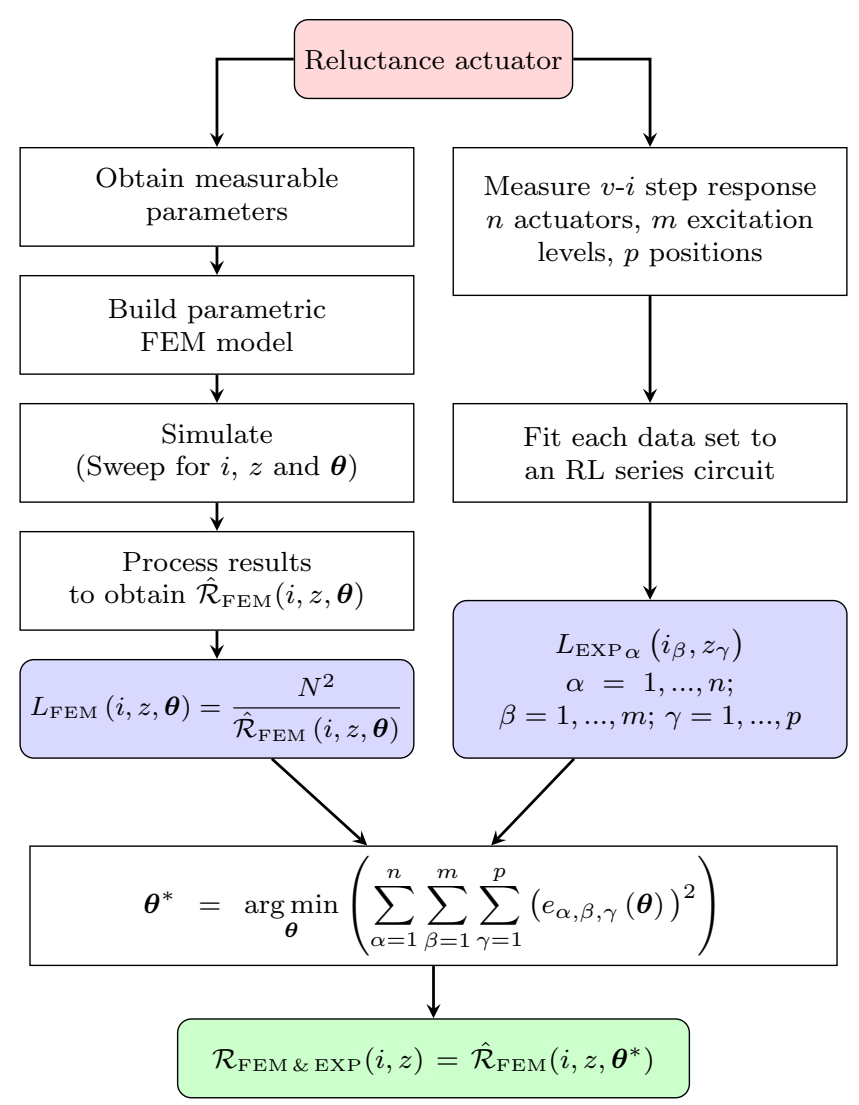

Figure 1: Flow chart of the proposed FEM \& EXP method

\section{Validation example}

The device used to validate the FEM \& EXP method is a commercial solenoid valve used for safety purposes in low-pressure gas lines (see Fig. 2). In this valve, the coil is wrapped around a cylindrical steel core which has a fixed and a movable part. The housing, which is also made of the same material, provides a low-reluctance return path for the flux. A helical spring ensures that the mechanism returns to its original position when the coil is de-energized. As a commercial device, this valve is sold by the manufacturer without providing much information about its design. Hence, although the geometry can be obtained from measurements, other parameters of the device are partially or completely unknown. In particular, we know neither the material of the core, which is some type of carbon steel, nor the number of turns of the coil. 


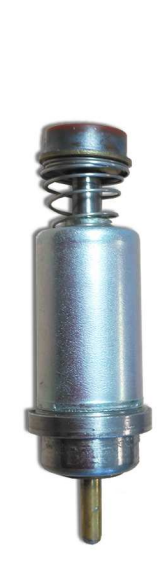

(a)

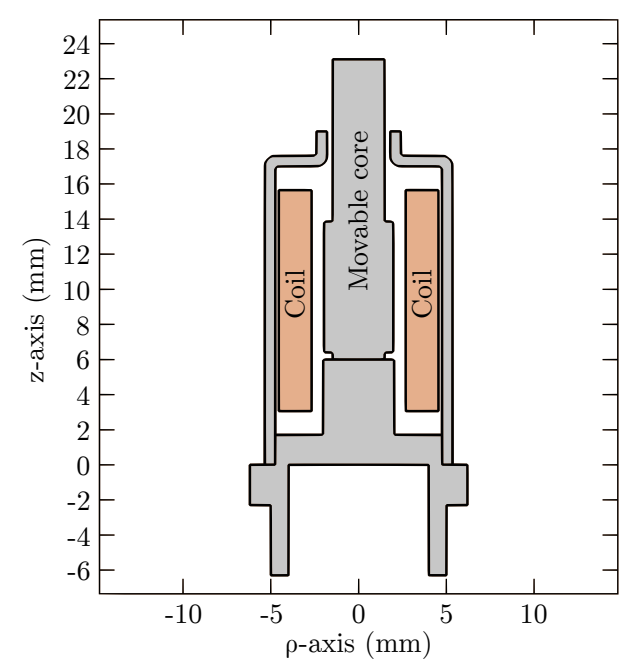

(b)
Figure 2: (a) Solenoid valve and (b) its geometry.

\subsection{Finite Element Model}

An FEM model has been built using the software COMSOL Multiphysics. The rotational symmetry of the valve has allowed for using a two-dimensional axisymmetric model, with a much lower computational complexity than its threedimensional equivalent. The coil has been modeled as a single-turn coil of rectangular section with uniform current density and total current $N i$. This simplification is widely used in the simulation of inductors [28, reduces significantly the modeling stage and the simulation time, and is magnetically equivalent to modeling all the turns of the coil. The material of the core is assumed magnetically linear, which causes $\mathcal{R}_{\text {FEM }}$ to be independent of the model excitation and permits using $(9)$ to compute its value.

Since the magnetic permeability of the core is unknown, the reluctance has been computed for seven different values, covering the complete range of usual values of ferromagnetic materials. For simplicity, instead of using the absolute permeability of the core, $\mu_{c}$, we will work with the relative value, $\mu_{r_{c}}=\mu_{c} / \mu_{0}$, where $\mu_{0}$ is the vacuum magnetic permeability. The highest selected value, which is $\mu_{r_{c}}=10^{6}$, has been used as an approximation of infinite magnetic permeability, i.e., the reluctance of the core in this case may be considered negligible and, consequently, $\mathcal{R}_{\mathrm{FEM}}$ only consists of the air gap component. On the other hand, the gap length, $z$, has been discretized into twenty different values between zero and the maximum gap $(z=3.35 \mathrm{~mm})$, using a non-uniform distribution with more values near the lower and upper bounds. A total of 140 FEM simulations have been consequently performed, taking approximately 50 minutes of computation time on a fourth generation Intel i7 processor. As an example, Fig. 3 shows the magnetic flux density norm in the valve obtained in three of these simulations.

The result of the process is therefore a 140 values lookup table of the reluctance as a function of the gap length and

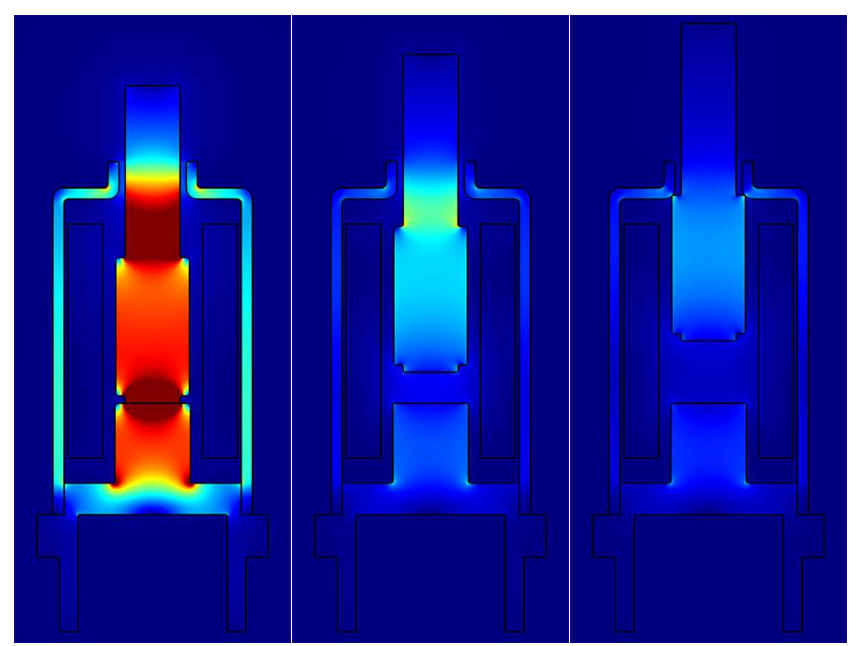

Figure 3: Magnetic flux density norm obtained from FEM simulations with $\mu_{r_{c}}=100$ at gap lengths of $0,1.675$, and $3.350 \mathrm{~mm}$, respectively. $N i=30 \mathrm{~A}$. The color scale goes from dark blue for $0 \mathrm{~T}$ to dark red for $125 \mathrm{mT}$.

the relative magnetic permeability of the core, $\mathcal{R}_{\mathrm{FEM}}\left(z, \mu_{r_{c}}\right)$. The obtained values are represented in Fig. 4. As shown, $\mathcal{R}_{\text {FEM }}$ begins with a value which corresponds to zero gap and, accordingly to (3), is higher the lower the magnetic permeability of the core. In this regard, note that the initial reluctance for $\mu_{r_{c}}=10^{6}$ is not zero although the core can be assumed perfectly magnetically permeable; this is due to the existence of a secondary annular air gap between the housing and the movable core (see Fig. 2b). Since the air is a low permeable material compared to the core, the reluctance increases with the gap length. The trend is similar for all the studied values of magnetic permeability. However, it is noteworthy that the derivative of $\mathcal{R}_{\mathrm{FEM}}$ with respect to $z$, which is one of the factors of the magnetic force (10), is highly different for gap lengths close to the limit values. This can be seen in Fig. 5, where this variable is represented as a function of the magnetic permeability for the minimum, middle and maximum gaps. It is particularly worth noting that, for the maximum gap, this derivative even changes sign for permeabilities greater than 200, which is due to the attraction force produced in the secondary air gap. The FEM based inductance per square turn, also known as permeance, is also represented in Fig. 6.

\subsection{Experimental identification}

As stated in Section 3 , the first step of the identification consists in measuring the inductance of the valve at several positions of the plunger. For that, it has been supplied with a square wave voltage between 0 and $0.45 \mathrm{~V}$, and the electric current through the coil has been measured with a shunt resistor. A Picoscope 4824 oscilloscope/signal generator has been used both to create the input and to record the output signals. The valve has been positioned at the desired gap lengths by means of a micrometric screw (see Fig. 7), and the tests have been conducted at low-level 


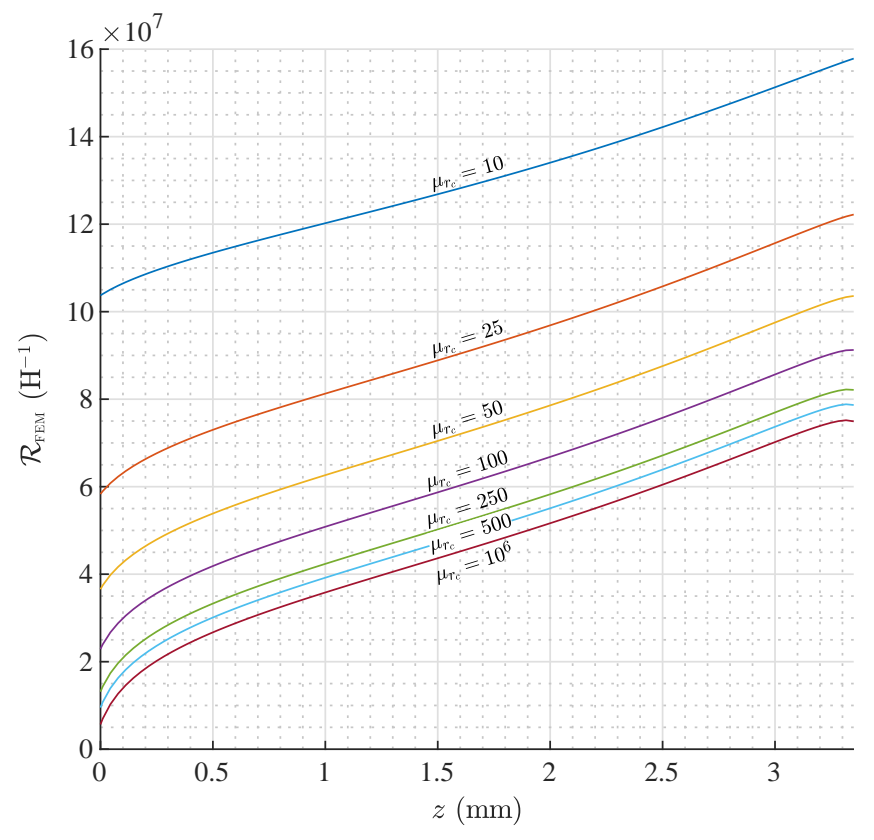

Figure 4: Reluctance obtained from FEM simulations as a function of gap length and of the core relative permeability.

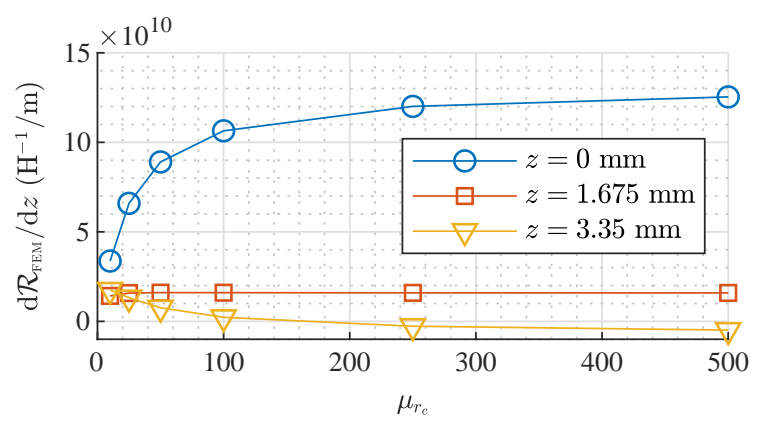

Figure 5: Derivative of the reluctance with respect to the gap length as a function of the relative permeability of the core. Values for gap lengths of $0,1.675$, and $3.35 \mathrm{~mm}$.

voltages so that the magnetic force generated was not high enough to move the plunger. Thus, the returning spring of the device maintains the desired position during the test.

In this case, the resulting FEM \& EXP model will be used to predict the dynamic behavior of any manufactured valve. Hence, a total of 68 tests have been conducted, which correspond to four different valves at 17 different positions. Then, a series RL linear model has been fitted to each one of the 68 input-output data sets, obtaining as a result the experimental values of resistance, $R_{\operatorname{EXP} \alpha}\left(z_{\gamma}\right)$, and inductance, $L_{\operatorname{EXP} \alpha}\left(z_{\gamma}\right)$, for $\alpha=1, \ldots, 4$ and $\gamma=1, \ldots, 17$. As an example, Fig. 8 shows the experimental response of the electric current for one of the valves at three different positions and the corresponding best-fitting RL model simulations.

Once the experimental inductance has been measured, the next step is to identify the best-fitting values of $\mu_{r_{c}}$ and $N$ by minimizing the performance index $J$. The optimization has been performed by means of the simplex search

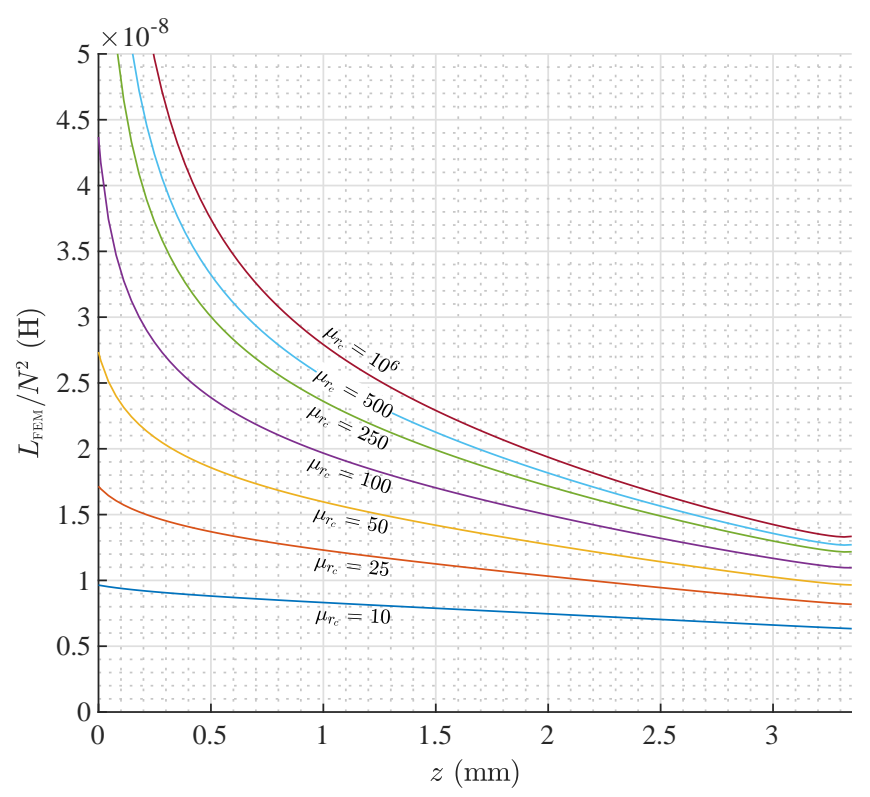

Figure 6: Inductance per square turn obtained from FEM simulations as a function of gap length and of the core relative magnetic permeability.

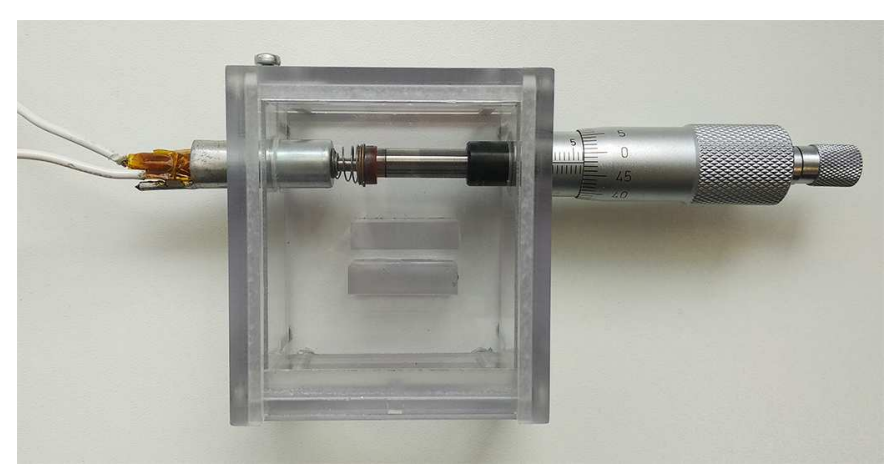

Figure 7: Prototype used in the experiments. A micrometric screw allows for a precise positioning of the plunger.

algorithm of Lagarias et al. 29], using as initial values $\mu_{r_{c}}=100$, which is a standard value for carbon steels, and $N=1000$, which is an approximation obtained from the ohmic resistance and the dimensions of the coil. The best-fitting parameter values found by the algorithm are $\mu_{r_{c}}{ }^{*}=89.13$ and $N^{*}=1178$, and the RMSE of the model with respect to the measurements is $1.06 \mathrm{mH}$. Considering that the experimental values of inductance already have an average standard deviation of $0.804 \mathrm{mH}$, we conclude that the fitting of the model is very good. Another value of the number of turns, $N=1207$, has been experimentally obtained from one specific valve by means of a winding machine with a built-in turn counter. Since $N^{*}$ is very similar to this value, we can state that the results of the proposed procedure are satisfactory. Besides, measuring $N$ by means of the winder implies destroying the device, so our proposal is clearly advantageous in this aspect.

Additional results of both identification stages are pre- 


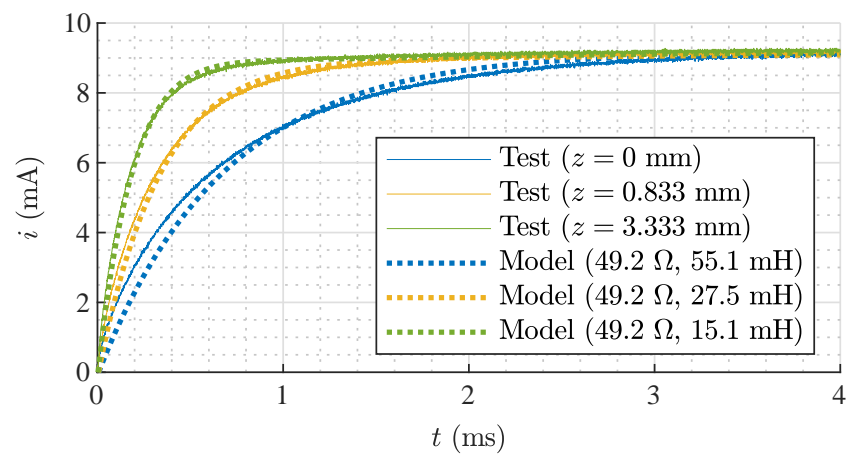

Figure 8: Electric current response to a $0.45 \mathrm{~V}$ voltage step. Experimental tests at three different gap lengths and best-fitting RL model simulations.

sented in Figs. 9 to 11 . In the first place, Fig. 9 shows the experimental resistance of the valve obtained from the RL model fitting. As expected, there is no evident dependence on the gap length for any of the four units. However, there are small but noticeable differences between devices which may be due to the inherent variations in the manufacturing process. Note also that the variability found on each device is perfectly explained by small temperature fluctuations between tests. Secondly, Fig. 10 shows the experimental inductances of the four valves as well as the FEM \& EXP model inductance, $L_{\mathrm{FEM} \& \mathrm{EXP}}(z)=\left(N^{*}\right)^{2} / \mathcal{R}_{\mathrm{FEM} \& \mathrm{EXP}}(z)$. In this case, the dependence on $z$ is evident and similar for all the units. Besides, it is shown that the model inductance fits very well with the experimental values. In this connection, Fig. 11 represents the residuals of the model, which are calculated as $r_{\alpha}=L_{\mathrm{EXP} \alpha}-L_{\mathrm{FEM} \& \mathrm{EXP}}$. It can be seen that, except for $z=0 \mathrm{~mm}$, these residuals present an evident tendency, which means that they are probably not due to experimental errors but to small model deviations. Nevertheless, the residuals are very small compared to the inductance, so we conclude that the identification procedure has been successful. With respect to the first position, note that this corresponds to the situation of a perfectly closed gap, which can be modeled but is hardly reachable in practice since surface irregularities are always present.

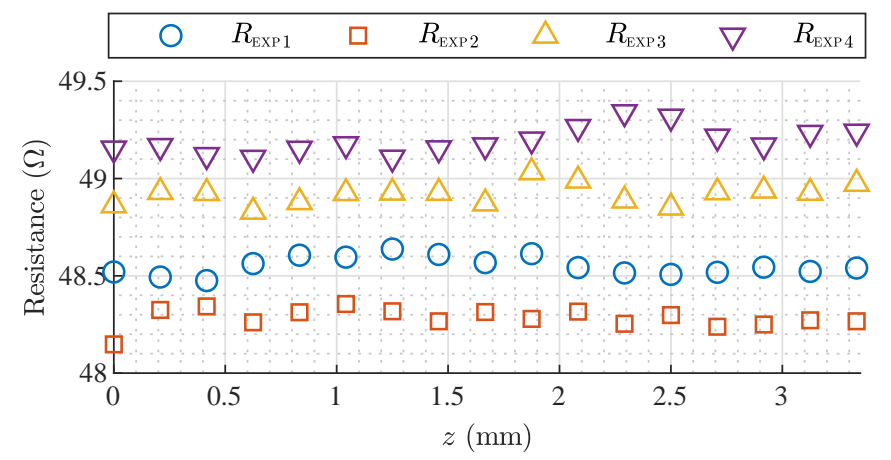

Figure 9: Experimental values of resistance as a function of gap length.

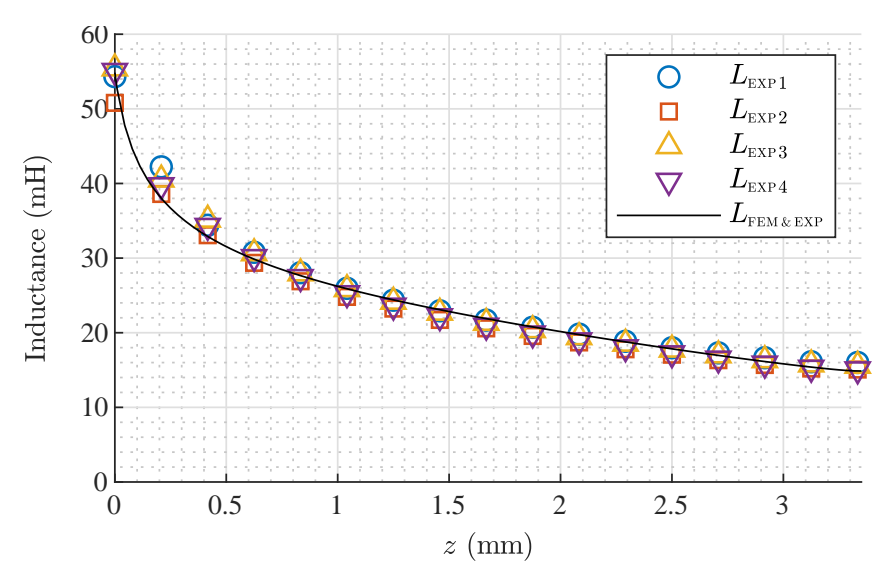

Figure 10: Experimental and FEM \& EXP values of inductance as a function of gap length.

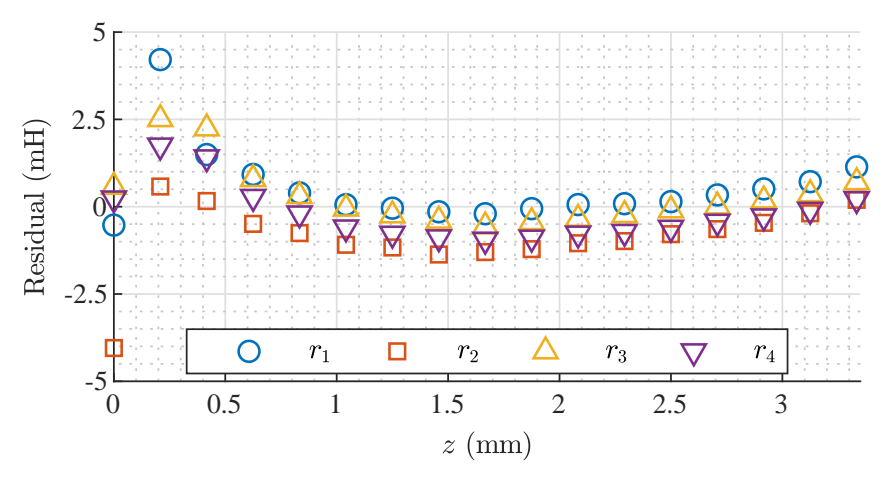

Figure 11: Residuals of the model as a function of gap length.

\subsection{Discussion on magnetic linearity}

Throughout the validation example we have assumed that the core material is magnetically linear. Under this assumption, the reluctance is independent of the model excitation and can be easily computed using the magnetic energy. In addition, the simulation time is greatly reduced for a twofold reason: the FEM model is linear and the parametric sweep is one dimension smaller. If desired, magnetic saturation could be included as explained in Section 3 i.e., by means of a parametric model. In that case, both the FEM simulations and the experimental tests would have to be carried out for several excitation levels so that the saturation effects are properly characterized.

Alternatively, magnetic saturation may also be modeled using an approximate method which does not require additional (and nonlinear) FEM simulations. Assuming that permeability only depends on the excitation current, the described identification procedure could be performed several times using small-signal measurements from tests at different levels of current. Thus, a family of reluctance and inductance curves would be obtained and the dependence of permeability on current could be studied. In this connection, the current during the step-response tests of the previous example varied approximately between 0 and $9 \mathrm{~mA}$ (see Fig. 8), so we can state that the obtained results correspond to a current of about $4.5 \mathrm{~mA}$. 


\section{Model comparison}

In this section, we compare our modeling method with other three common approaches in the literature:

- First, as proposed in some works [21, a model whose reluctance is assumed linear with respect to the gap length. In this case, this linear reluctance has been fitted to $\mathcal{R}_{\text {FEM \& EXP }}$ by least squares and the corresponding inductance has been computed by (11) using the already identified value of $N^{*}=1178$.

- Secondly, a purely numerical model similar to those presented in [16] or [17]. For that, we directly use the results from the presented FEM model for $\mu_{r_{c}}=100$ and $N=1000$, which were the initial guesses for the unknown parameters and, hence, are the logical values to use if no experiments have been performed.

- Finally, a theoretical model using the MEC approach as in [19] or [20]. For that, the magnetic circuit has been decomposed in several elements of simple geometry (see Fig. 12) whose reluctances can be analytically calculated. Specifically, all the resulting elements have been approximated as cylinders or cylindrical rings whose dimensions and reluctances are shown in Table 1. Note that, in order to account for flux fringing effects, the reluctance of the primary air gap incorporates the correction factor proposed by McLyman 30.
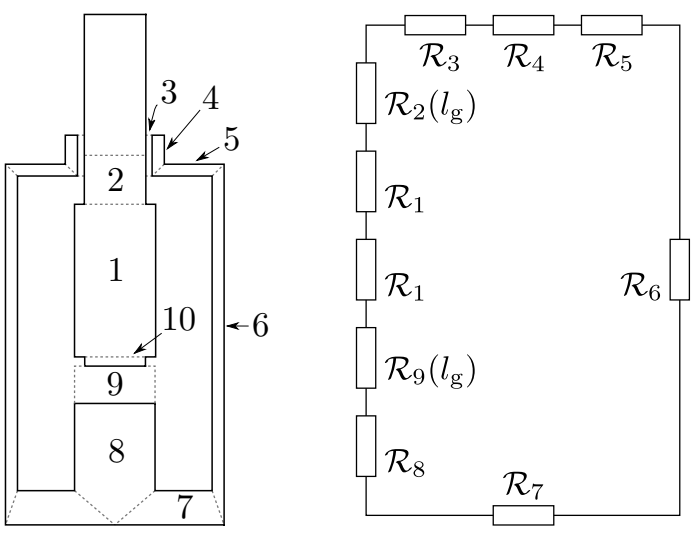

Figure 12: Magnetic circuit of the valve and reluctance decomposition. Reluctances $\mathcal{R}_{2}$ and $\mathcal{R}_{9}$ depend on the gap length, $z$.

The reluctance values obtained from the four different approaches are presented in Fig. 13. As shown, a linear reluctance could be used to link magnetic flux and electric current with little error. Actually, the root-mean-square deviation (RMSD) between both models has a value of $1.38 \cdot 10^{6} \mathrm{H}^{-1}$, which represents only about $2 \%$ of the mean value of $\mathcal{R}_{\text {FEM \& Exp. Since the identified value of }}$ permeability, $\mu_{r_{c}}{ }^{*}=89.13$, is close to the initial guess of $\mu_{r_{c}}=100$, the FEM reluctance is also very similar and has a RMSD of about 3\%. On the other hand, the MEC reluctance is less accurate, basically because of the simplified geometry and the assumptions of the method, and the RMSD in this case reaches about $18 \%$ of the mean value of the reluctance. Table 2 summarizes the RMSD of the methods.

The inductance values are represented in Fig. 14. These results, together with the experimental fitting of Fig. 10 . show clearly that the studied approaches are not as accurate as our proposal. In this regard, the RMSE of the models with respect to the experimental values of inductance are $3.29 \mathrm{mH}$ for the linear reluctance method, $6.41 \mathrm{mH}$ for the FEM model and $11.3 \mathrm{mH}$ for the MEC approach, all of them much higher than the $1.06 \mathrm{mH}$ error of the FEM \& EXP method (see Section 4.2). It is noteworthy that, while the linear reluctance model provides a very accurate inductance except for small gap lengths, the difference between the estimated and the identified values of the number of turns causes the FEM and MEC models to have much greater errors.

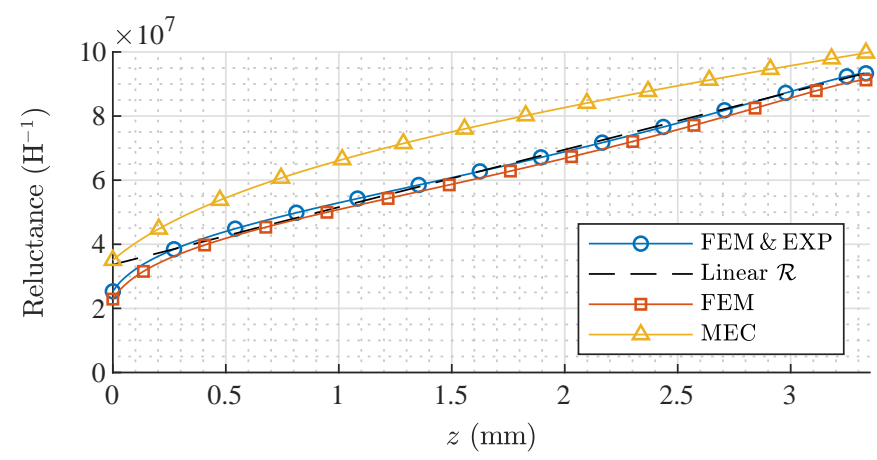

Figure 13: Reluctance as a function of gap length. Method comparison.

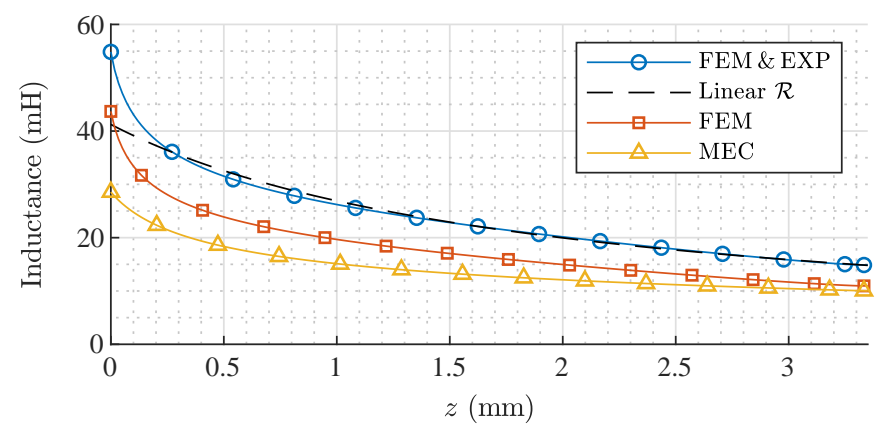

Figure 14: Inductance as a function of gap length. Method comparison.

The reluctance of the device is also related to the magnetic force that drives the motion. Given 10 , this force may be studied, e.g., per square unit of flux,

$$
F_{m a g} / \phi^{2}=-\frac{1}{2} \frac{\mathrm{d} \mathcal{R}}{\mathrm{d} z}
$$

which allows for analyzing the errors in the derivative of the reluctance with respect to the gap length, or per square 
Table 1: Reluctance decomposition of the MEC model. Each element has internal radius $R_{\text {int }}$, external radius $R_{\text {out }}$ and height $h$.

\begin{tabular}{llllllll}
\hline Reluctance & Geometry & Flux Direction & $\mu$ & $R_{\text {int }}(\mathrm{mm})$ & $R_{\text {out }}(\mathrm{mm})$ & $h(\mathrm{~mm})$ & Value $(\mathrm{A} / \mathrm{Wb})$ \\
\hline $\mathcal{R}_{1}$ & Cylinder & Axial & $100 \mu_{0}$ & - & 2 & 7.45 & $4.72 \cdot 10^{6}$ \\
$\mathcal{R}_{2}$ & Cylinder & Axial & $100 \mu_{0}$ & - & 1.5 & $4.15-z[\mathrm{~mm}]$ & $1.13 \cdot 10^{6} \cdot(4.15-z[\mathrm{~mm}])$ \\
$\mathcal{R}_{3}$ & Ring & Radial & $100 \mu_{0}$ & 1.5 & 1.8 & 2 & $1.15 \cdot 10^{7}$ \\
$\mathcal{R}_{4}$ & Ring & Radial & $\mu_{0}$ & 1.8 & 2.4 & 1.7 & $2.14 \cdot 10^{5}$ \\
$\mathcal{R}_{5}$ & Ring & Radial & $100 \mu_{0}$ & 2.1 & 5.05 & 0.6 & $1.85 \cdot 10^{6}$ \\
$\mathcal{R}_{6}$ & Ring & Axial & $100 \mu_{0}$ & 4.75 & 5.35 & 17 & $7.11 \cdot 10^{6}$ \\
$\mathcal{R}_{7}$ & Ring & Radial & $100 \mu_{0}$ & 1 & 5.05 & 1.7 & $1.21 \cdot 10^{6}$ \\
$\mathcal{R}_{8}$ & Cylinder & Axial & $100 \mu_{0}$ & - & 2 & 5.15 & $3.26 \cdot 10^{6}$ \\
$\mathcal{R}_{9}$ & Cylinder & Axial & $\mu_{0}$ & - & 2 & $z .33 \cdot 10^{7} \cdot z[\mathrm{~mm}]$ \\
& & & & & & & $\frac{z}{z[\mathrm{~mm}]}$ \\
$\mathcal{R}_{10}$ & Cylinder & Axial & $100 \mu_{0}$ & - & 1.5 & 0.4 & 3.54 \\
\hline
\end{tabular}

Table 2: Root-mean-square deviations between the FEM \& EXP modeling method and the most common approaches in literature. In parentheses, magnitude of the errors with respect to the mean absolute value of the corresponding variable.

\begin{tabular}{lcccc}
\hline \multirow{2}{*}{ Modeling method } & \multicolumn{4}{c}{ Variable } \\
\cline { 2 - 5 } & $\mathcal{R}$ & $L$ & $\left|F_{\text {mag }}\right| / \phi^{2}$ & $\left|F_{\text {mag }}\right| / i^{2}$ \\
\hline Linear $\mathcal{R}$ & $1.38 \cdot 10^{6} \mathrm{H}^{-1}(2.17 \%)$ & $1.67 \mathrm{mH}(7.01 \%)$ & $5.33 \cdot 10^{9} \mathrm{~N} / \mathrm{Wb}^{2}(52.4 \%)$ & $10.2 \mathrm{~N}^{2} \mathrm{~A}^{2}(167 \%)$ \\
FEM & $2.09 \cdot 10^{6} \mathrm{H}^{-1}(3.28 \%)$ & $6.14 \mathrm{mH}(25.8 \%)$ & $1.52 \cdot 10^{8} \mathrm{~N} / \mathrm{Wb}^{2}(1.49 \%)$ & $1.75 \mathrm{~N} / \mathrm{A}^{2}(28.7 \%)$ \\
MEC & $1.17 \cdot 10^{7} \mathrm{H}^{-1}(18.4 \%)$ & $10.4 \mathrm{mH}(43.5 \%)$ & $3.02 \cdot 10^{9} \mathrm{~N} / \mathrm{Wb}^{2}(29.8 \%)$ & $8.59 \mathrm{~N} / \mathrm{A}^{2}(141 \%)$ \\
\hline
\end{tabular}

unit of current,

$$
F_{\text {mag }} / i^{2}=-\frac{1}{2} \frac{N^{2}}{\mathcal{R}^{2}} \frac{\mathrm{d} \mathcal{R}}{\mathrm{d} z}
$$

which provides a more integrated analysis because it includes the errors in the reluctance, its derivative, and also in the number of turns of the coil. Hence, both options have been considered and the results are presented in Figs. 15 and 16 and also in Table 2 . In order to see clearly the differences among methods, the graphs show separately the magnetic force for small lengths of the gap, $z \in[0,0.4] \mathrm{mm}$, and for the rest of possible values of $z$. As shown, the magnetic force calculated with the FEM \& EXP model has its maximum absolute value for $z=0$ and decreases rapidly with the gap length. Besides, it becomes almost zero for the maximum gap, which indicates that the electromagnet can not pull the mechanism if it has been completely released. On the other hand, it is evident that the force resulting from the linear $\mathcal{R}$ does not reproduce accurately the actual behavior, especially for low and high values of the gap. Actually, the RMSDs of this method reach about $52 \%$ and $167 \%$ of their respective mean absolute values, which definitely proves that it should not be used to compute the force, even if the number of turns of the coil is perfectly known. The reluctance derivative provided by the MEC model is slightly better, but the best approximation is given by the FEM model, whose RMSD is only about $1.5 \%$. In any case, the error in the initial guess of $N$ makes these two approaches reach very high errors when evaluating $F_{\text {mag }} / i^{2}$.

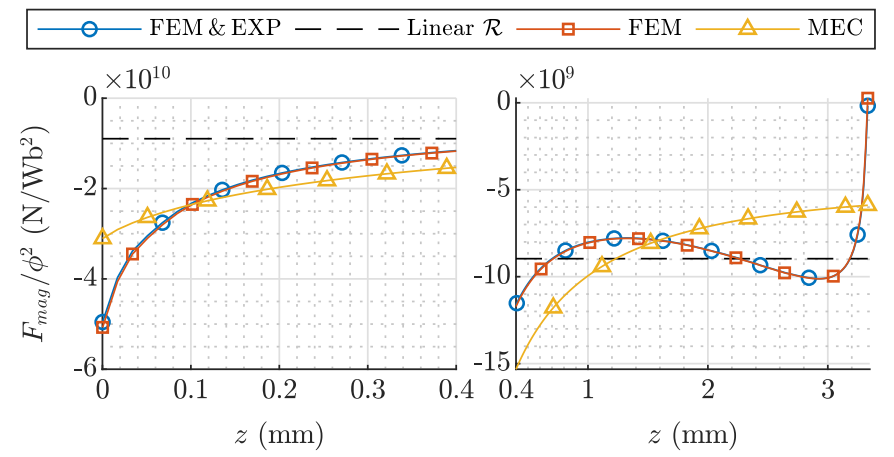

Figure 15: Magnetic force per square unit of flux as a function of gap length.

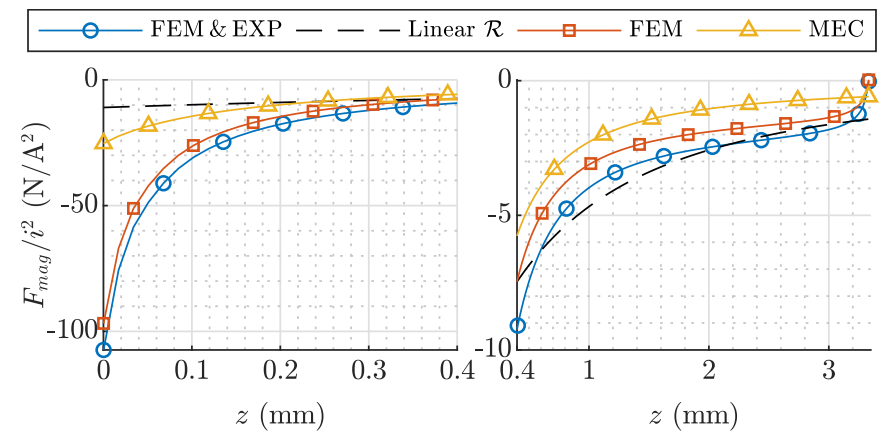

Figure 16: Magnetic force per square unit of current as a function of gap length. 


\section{Conclusions}

As shown, describing accurately the reluctance of any electromechanical device is critical to analyze its dynamics. In this paper we have presented a new method for characterizing the reluctance of a commercial device by combining results from FEM simulations and data acquired in experimental tests. This fusion permits overcoming the drawbacks that both approaches have separately, i.e., the lack of information about some aspects of the system or the assumption of too many simplifications. In this regard, the FEM model allows for a precise study of the reluctance with respect to the position of the mechanism, while the identification procedure makes use of experimental data to determine the unknown parameters.

Once the properties of the device are identified and the reluctance completely characterized, the magnetic force can also be obtained and analyzed. We have seen that, for a given value of current or of magnetic flux, this force is maximum for zero gap and decreases rapidly with the position during the first stage of the motion. A further discussion has shown that a linear approximation of the reluctance with respect to the gap length could be used to relate current and magnetic flux, but it would lead to high errors if used to calculate the magnetic force. Besides, MEC and FEM models have been also included in the comparison and we have shown that the errors with our approach are between five and ten times smaller with respect to the inductance.

The method has been illustrated by an example with a solenoid valve which had two unknown parameters: the number of turns of the coil and the magnetic permeability of the core. However, the procedure can also be used if more parameters of the device have to be identified. Furthermore, it is fully applicable to any other variable reluctance device such as relays or any type of short-stroke actuator. In this regard, the resulting models are expected to lead to more precise simulations and to better control and estimation algorithms.

\section{Acknowledgments}

This work was supported by the Ministerio de Economía y Competitividad, Gobierno de España - European Union, under project RTC-2014-1847-6, the Ministerio de Educación, Cultura y Deporte, Gobierno de España, under grant FPU14/04171, and by project DGA-T45_17R/FSE.

\section{References}

[1] M. Branciforte, A. Meli, G. Muscato, D. Porto, Ann and noninteger order modeling of abs solenoid valves, IEEE Trans. Control Syst. Technol. 19 (3) (2011) 628-635.

[2] J. Zhao, R. J. Seethaler, Compensating combustion forces for automotive electromagnetic valves, Mechatronics 20 (4) (2010) $433-441$.
[3] Y. P. Yang, J. J. Liu, D. H. Ye, Y. R. Chen, P. H. Lu, Multiobjective optimal design and soft landing control of an electromagnetic valve actuator for a camless engine, IEEE/ASME Trans. Mechatronics 18 (3) (2013) 963-972.

[4] M. Naidu, S. Gopalakrishnan, T. Nehl, Fault-tolerant permanent magnet motor drive topologies for automotive x-by-wire systems, IEEE Trans. Ind. Appl. 46 (2) (2010) 841-848.

[5] J. Acero, J. Burdio, L. Barragan, D. Navarro, R. Alonso, J. Ramon, F. Monterde, P. Hernandez, S. Llorente, I. Garde, Domestic induction appliances, IEEE Ind. Appl. Mag. 2 (16) (2010) 39-47.

[6] S. Haghbin, S. Lundmark, M. Alaküla, O. Carlson, Gridconnected integrated battery chargers in vehicle applications: Review and new solution, IEEE Trans. Ind. Electron. 60 (2) (2013) 459-473.

[7] D. Marcsa, M. Kuczmann, Design and control for torque ripple reduction of a 3-phase switched reluctance motor, Computers \& Mathematics with Applications 74 (1) (2017) 89 - 95.

[8] T. van der Sande, B. Gysen, I. Besselink, J. Paulides, E. Lomonova, H. Nijmeijer, Robust control of an electromagnetic active suspension system: Simulations and measurements, Mechatronics 23 (2) (2013) 204 - 212, special Issue on Linear Drives.

[9] P. Enrici, F. Dumas, N. Ziegler, D. Matt, Design of a highperformance multi-air gap linear actuator for aeronautical applications, IEEE Trans. Energy Convers. 31 (3) (2016) 896-905.

[10] G. Bramerdorfer, A. C. Zavoianu, S. Silber, E. Lughofer, W. Amrhein, Possibilities for speeding up the fe-based optimization of electrical machines - a case study, IEEE Trans. Ind. Appl. 52 (6) (2016) 4668-4677.

[11] P. Eyabi, G. Washington, Modeling and sensorless control of an electromagnetic valve actuator, Mechatronics 16 (3) (2006) 159 $-175$.

[12] J. Tsai, C. R. Koch, M. Saif, Cycle adaptive feedforward approach controllers for an electromagnetic valve actuator, IEEE Trans. Control Syst. Technol. 20 (3) (2012) 738-746.

[13] P. Mercorelli, An antisaturating adaptive preaction and a slide surface to achieve soft landing control for electromagnetic actuators, IEEE/ASME Trans. Mechatronics 17 (1) (2012) 76-85.

[14] J.-H. Lee, Y.-W. Yun, H.-W. Hong, M.-K. Park, Control of spool position of on/off solenoid operated hydraulic valve by sliding-mode controller, J. Mech. Sci. Technol. 29 (12) (2015) 5395-5408.

[15] E. Ramirez-Laboreo, C. Sagues, S. Llorente, A new run-torun approach for reducing contact bounce in electromagnetic switches, IEEE Trans. Ind. Electron. 64 (1) (2017) 535-543.

[16] J. Bao, N. H. Vrijsen, B. L. J. Gysen, R. L. J. Sprangers, E. A. Lomonova, Optimization of the force density for medium-stroke reluctance actuators, IEEE Trans. Ind. Appl. 50 (5) (2014) 3194-3202.

[17] B. Xu, R. Ding, J. Zhang, L. Sha, M. Cheng, Multiphysicscoupled modeling: Simulation of the hydraulic-operating mechanism for a sf6 high-voltage circuit breaker, IEEE/ASME Trans. Mechatronics 21 (1) (2016) 379-393.

[18] A. di Gaeta, U. Montanaro, V. Giglio, Experimental validation of a hybrid analytical-fem model of an electromagnetic engine valve actuator and its control application, IEEE/ASME Trans. Mechatronics 18 (2) (2013) 807-812.

[19] S. Fang, Q. Liu, H. Lin, S. L. Ho, A novel flux-weakening control strategy for permanent-magnet actuator of vacuum circuit breaker, IEEE Trans. Ind. Electron. 63 (4) (2016) 2275-2283.

[20] W. Kemmetmueller, D. Faustner, A. Kugi, Optimal torque control of permanent magnet synchronous machines using magnetic equivalent circuits, Mechatronics 32 (2015) 22 - 33.

[21] E. Ramirez-Laboreo, C. Sagues, S. Llorente, A new model of electromechanical relays for predicting the motion and electromagnetic dynamics, IEEE Trans. Ind. Appl. 52 (3) (2016) 25452553.

[22] Z. Guofo, W. Qiya, R. Wanbin, An output space-mapping algorithm to optimize the dimensional parameter of electromagnetic relay, IEEE Trans. Magn. 47 (9) (2011) 2194-2199. 
[23] D. Wattiaux, O. Verlinden, Modelling of the dynamic behaviour of electromechanical relays for the analysis of sensitivity to shocks and vibrations, Exp. Mech. 51 (9) (2011) 1459-1472.

[24] B. L. J. Gysen, K. J. Meessen, J. J. H. Paulides, E. A. Lomonova, General formulation of the electromagnetic field distribution in machines and devices using fourier analysis, IEEE Trans. Magn. 46 (1) (2010) 39-52.

[25] H. Nouri, N. Larsen, T. Davies, Contact bounce simulation using matlab, in: Proc. 43rd IEEE Holm Conf. Elect. Contacts, IEEE, 1997, pp. 284-288.

[26] X. Jun, H. Jun-jia, Z. Chun-yan, A dynamic model of electromagnetic relay including contact bounce, in: 2008 Int. Conf. Elect. Mach. and Syst., IEEE, 2008, pp. 4144-4149.

[27] L. Ljung, System Identification - Theory for the User, 2nd Edition, Prentice-Hall, Upper Saddle River, N.J, 1999.

[28] I. Lope, J. Acero, C. Carretero, Analysis and optimization of the efficiency of induction heating applications with litz-wire planar and solenoidal coils, IEEE Trans. Power Electron. 31 (7) (2016) 5089-5101.

[29] J. C. Lagarias, J. A. Reeds, M. H. Wright, P. E. Wright, Convergence properties of the nelder-mead simplex method in low dimensions, SIAM J. Optim. 9 (1) (1998) 112-147.

[30] C. W. T. McLyman, Transformer and inductor design handbook, CRC press, 2016. 